\title{
Association of Polar Amino Acids at Position 26 of the HLA-DQB1 First Domain with the Anticentromere Autoantibody Response in Systemic Sclerosis (Scleroderma)
}

\author{
John D. Reveille," David Owerbach," Rose Goldstein," Ramon Moreda," Reuben A. Isem, " and Frank C. Arnett* \\ *Division of Rheumatology and Clinical Immunogenetics, Department of Internal Medicine, The University of Texas Medical School at \\ Houston, Houston, Texas 77225; ${ }^{\ddagger}$ Division of Endocrinology and Metabolism, Department of Pediatrics, Baylor College of Medicine, \\ Houston, Texas 77030; 'Division of Rheumatology, Department of Internal Medicine, Ottawa General Hospital, \\ The University of Ottawa, Ottawa, Canada; "Division of Rheumatology, Department of Internal Medicine, \\ The University of Miami School of Medicine, Miami, Florida 33010
}

\begin{abstract}
HLA class II alleles (detected by DNA typing) were determined in 116 Caucasians with systemic sclerosis positive and negative for anticentromere autoantibodies (ACA). Significantly increased frequencies of HLA-DR5(DRw11) ( $P$ $=0.009)$ and the Dw13(DRB1*0403, *0407) subtypes of DR4 (probability corrected, $P c=0.005$ ) were seen in ACA positive patients, and HLA-DR1 and DRw8 were also increased. These findings appeared to reflect linkage disequilibrium of DR5(DRw11) and many DR4(Dw13) haplotypes with HLADQw7 and DR1 with DQw5. In fact, the presence of a DQB1 allele having a polar glycine or tyrosine at position 26 of the DQB1 first domain versus a hydrophobic leucine accounted for $100 \%$ of ACA positive Caucasian systemic sclerosis patients compared to $69 \%$ of the ACA negative SS patients $(P=0.0008)$ and $71 \%$ of Caucasian controls $(P=\mathbf{0 . 0 0 0 3})$ as well as all 7 ACA patients of non-Caucasian background. Furthermore, the genotype frequency of DQB1 alleles lacking leucine at position 26 was $73 \%$ in ACA positive SS patients, compared to $42 \%$ of ACA negative patients $\left(P=1.2 \times 10^{-5}\right)$ and $38 \%$ of controls $(P$ $\left.=5.8 \times 10^{-7}\right)$. These data, then, suggest that the second hypervariable region of the HLA-DQB1 chain may form the candidate epitope associated with the ACA response. (J. Clin. Invest. 1992. 89:1 208-1213.) Key words: immunogenetics • autoimmunity $\bullet$ nucleoprotein $\bullet$ genotype $\bullet$ autoantigen
\end{abstract}

\section{Introduction}

Systemic sclerosis $(\mathrm{SS})^{1}$ or scleroderma encompasses a wide clinical spectrum with disease severity ranging from patients only with Raynaud's phenomenon and minor skin changes, to

Corresponding address to John D. Reveille, M.D., Division of Rheumatology and Clinical Immunogenetics, University of Texas Medical School, P.O. Box 20708, Houston, TX 77225.

Received for publication 9 July 1991 and in revised form 15 October 1991.

1. Abbreviations used in this paper: ACA, anticentromere antibodies; $\mathrm{OR}$, odds ratio; $P c$, probability corrected; PCR, polymerase chain reaction; RFLP, restriction fragment length polymorphism; SS, systemic sclerosis.

J. Clin. Invest.

(c) The American Society for Clinical Investigation, Inc.

0021-9738/92/04/1208/06 \$2.00

Volume 89, April 1992, 1208-1213 those with widespread systemic fibrosis with critical pulmonary, cardiac, gastrointestinal, and renal involvement. SS is also a serologically heterogeneous disease with several highly disease-specific autoantibodies associating with certain clinical subsets. For example, anticentromere antibodies (ACA) are associated with limited skin and visceral involvement, including the CREST variant (subcutaneous calcinosis, Raynaud's phenomenon, esophageal dysmotility, sclerodactyly, and telangiectasia) which usually protends a better prognosis (1-8). Anti-topoisomerase I antibodies (anti-Scl-70) have been correlated with widespread skin and systemic disease (7-9). The presence of anti-PM-Scl, one of the anti-nucleolar antibodies, as well as anti-U1-RNP, have been associated with myositis, which occasionally occurs in SS patients (10).

While the cause of SS is unknown, both environmental and genetic factors have been implicated in pathogenesis. Ascertainment of the latter has resulted in a number of studies examining genetic markers, especially those encoded by genes of the major histocompatibility complex. A variety of studies have reported associations of SS with class I MHC specificities, including HLA-A9 $(11,12)$, B8 $(13,14)$, and B35 $(15,16)$; class II MHC, including HLA-DR1 (16-19), DR3 $(14,20)$ and DR5 (19, 21-23); and class III MHC, specifically C4 null alleles (15, 22). These associations have been generally weak and inconsistent between different centers, and some investigations have even been unable to show any significant HLA associations with SS (24-28).

A primary role of class II HLA molecules is the presentation of processed antigen to the $T$ cell receptor on helper $T$ lymphocytes resulting in antigen specific immune responses. Thus, specific autoantibody subsets of SS might be expected to show stronger correlations with class II MHC polymorphisms. Anti-topoisomerase I antibodies, for example, have been associated with HLA-DR2 (28) and DR5(DRw11) (8). An increased frequency of HLA-DR1 has been found in patients with ACA in some reports $(8,29)$. The latter of these two studies reported an association of ACA with HLA-DR1, DR4 and DRw8 (29), although many of the ACA positive patients were felt to have rheumatoid arthritis, an HLA-DR4-associated disease. Moreover, the HLA-DR1 correlation with ACA has not been confirmed by others $(28,30)$. Another recent study, using restriction fragment length polymorphism (RFLP) analysis, described an increased frequency of HLA-DR5(DRw11) in SS patients with ACA (23). HLA-DQw7 was also increased in this study but was felt to reflect linkage disequilibrium between HLA-DR5(DRw11) and DQw7.

In order to resolve these discrepancies and to ascertain the relative contributions of the three functional MHC class II gene 
families to the ACA response, we have here used RFLP, polymerase chain reaction (PCR) amplification, and DNA typing with oligonucleotide probes to examine the extensive polymorphism that has been recognized at the HLA-DR, DQ, and DP loci in a large, well-defined population of SS patients with and without ACA autoantibodies.

\section{Methods}

Patients and controls. As an ongoing study, all patients with systemic sclerosis (SS) seen by members of the Divisions of Rheumatology at the University of Texas Health Science Center at Houston (J. D. Reveille, R. A. Isern, F. C. Arnett), Ottawa General Hospital, the University of Ottawa (R. Goldstein) and the University of Miami School of Medicine (R. Moreda) are studied for a variety of autoantibodies and undergo HLA-DR, DQ, and DP typing by RFLP and/or oligotyping methods after informed consent is obtained. To date 156 patients with SS have been evaluated, including 67 from Houston, 35 from Ottawa and 41 from Miami, as well as 10 from Georgia who were part of an epidemiologic study reported previously (31). 116 were Caucasian, 27 American black, 11 Mexican or Central American, and 2 Asian. All patients had either SS by the preliminary criteria formulated by the American College of Rheumatology (32), or the CREST syndrome if at least three out of the five CREST symptoms were observed during the course of the disease.

Normal controls included 79 local Houston Caucasian volunteers who were blood donors or medical school personnel whose HLA-DR and DQ alleles were similarly defined by RFLP and oligotyping analyses. Most (85\%) had northwestern European surnames. Additional local blood donors known to be HLA-DR1, DR4 or DR5(DRw11) positive were also studied to ascertain reliable local frequencies of each of the DR1, DR4 and DR5(DRw11) subtypes.

Autoantibody determinations. Antinuclear antibodies were detected by indirect immunofluorescence on $\mathrm{HEp}-2$ cells with positivity defined as serum dilutions $\geq 1 / 80$. ACA was determined according to the typical immunofluorescent pattern on interphase and metaphase nuclei.

RFLP analysis of $H L A-D R$ and $D Q$ alleles. Genomic DNA was extracted from peripheral blood leukocytes (33); $10 \mu \mathrm{g}$ was digested with the restriction endonucleases BamH1 and TaqI under conditions specified by the manufacturer, and then electrophoresed through an $0.8 \%$ agarose gel overnight at $50 \mathrm{~V}$. The gel was then stained in ethidium bromide, photographed, subjected to denaturation and neutralization as described previously (34), and then transferred on to Zetabind membranes. (Cuno, Inc., Meriden, CT).

Probes used in this study included a $770 \mathrm{bp} \mathrm{Sacl/HindIII-cut} \mathrm{full-}$ length DRB1 cDNA (35), a 700 bp full-length PstI-cut DQB1 cDNA (36) and a $2.4 \mathrm{~kb}$ genomic PstI cut DQA1 probe (37) which were radiolabeled with $\alpha \mathrm{CTP}^{32}$ by oligolabeling (38). Prehybridization and hybridization conditions were carried out as previously described (34).

HLA-DR and DQ specificities were assigned to specific RFLP bands at the 10th International Histocompatibility Testing Workshop (IHTW) (39), as well as in our own $(40,41)$ and other's studies (42-46). Specific HLA-DQA1 and DQB1 chain alleles in each individual were assigned by comparison with reported DQA1 and DQB1 RFLP's for specific HLA-D haplotypes as determined by the World Health Organization Nomenclature Committee for Factors of the HLA System (47).

Oligotyping for $H L A-D R 1, D R 4$, and DR5 subtypes and $H L A$ $D Q A 1, D Q B 1$, and DPB1 alleles. For HLA-DR1 and DR5 subtyping, patients and controls positive for DR1 or DR5 by RFLP typing underwent group specific polymerase chain reaction (PCR) amplification of DRB1-DR1 alleles using the primers DRBAMP-1 (5'-TTCTTGTGGCAGCTTAAGTT-3) and DRAMP-B (5'-CCGCTGCACTGTGAAGCTCT-3') or DRB1-DR5 alleles using DRBAMP-3 (5'-CACGTTTCTTGGAGTACTCTAC-3') and DRBAMP-B. The PCR was carried out for 30 cycles under the following parameters: denaturation for 1 min at $96^{\circ} \mathrm{C}$, annealing $30 \mathrm{~s}$ at $60^{\circ} \mathrm{C}$, extension $2 \mathrm{~min}$ at $72^{\circ} \mathrm{C}$. Hybrid- ization and washing conditions were as has been recently reported elsewhere (48).

18-mer oligonucleotide probes, supplied by the 11th International Histocompatibility Testing Workshop, were used to detect polymorphic sequences around positions 70 and 86 of the DRB1 first domain to distinguish DRB1*0101, *0102 and ${ }^{*} 0103$ and DRB1*1101, ${ }^{*} 1102$, *1103 and *1104. HLA-DR4 subtypes were determined using primers described previously (49) as well as conditions and oligonucleotide probes previously published (50).

For HLA-DQA1 typing, genomic DNA was amplified with the primer pair DQAAMP-A (5-ATGGTGTAAACTTGTACCAGT-3') and DQAAMP-B (5'TTGGTAGCAGCGGTAGAGTTG-3') under conditions outlined for DRB, except the extension step for the PCR was for $1 \mathrm{~min}$ at $55^{\circ} \mathrm{C}$. Oligonucleotide probes corresponding to the polymorphisms at positions $25,34,55$, and 69 were used for the hybridizations as supplied by the 11 th International Histocompatibility Testing Workshop.

For HLA-DQB1 typing, the primers used included DQBAMP-A (5'CATGTGCTACTTCACCAACGG-3') and DQBAMP-B (5'-CTGGTAGTTGTGTCTGCACAC-3) under the same PCR conditions as the DQA1 primers. Oligonucleotide probes corresponding to the polymorphic codons $26,37,45,49,57$, and 70 were used for dot blot hybridization.

HLA-DPB1 typing was carried out with the primers DPBAMP-A (5'-GAGAGTGGCGCCTCCGCTCAT-3') and DPBAMP-B (5'-GCCGGCCCAAAGCCCTCACTC-3'), using the same PCR conditions as for HLA-DR5. 18-mer oligonucleotide probes corresponding to the polymorphic codons $9,35,55,69,76$, and 85 were used for the dot blot hybridizations.

Statistical analyses. Comparisons of HLA specificities and allelic frequencies in ACA positive verses ACA negative SS patients and versus normal race-matched controls were performed using the chi square analysis of $2 \times 2$ tables and Yates' correction with the EPI-INFO statistical program. Significant $P$ values were corrected $(P c)$ by multiplying the number of comparisons, i.e., for DR specificities $\times 12$, DR4-Dw subtypes $\times 5$, DQ specificities $\times 8$, DQA 1 alleles $\times 7$, DQB 1 alleles $\times 12$, and DPB1 alleles $\times 19$. Where an association with a particular specificity had been reported previously, the $P$ value was not corrected. Relative risks were calculated as odds ratios (OR).

\section{Results}

ACA were present in $42(36 \%)$ of the Caucasian SS patients, but were infrequent in patients of other races. Examination of HLA-DR specificities showed HLA-DR5 (DRw11) to be significantly increased in Caucasians with ACA compared to normal controls $(P=0.009, \mathrm{OR}=4)$ (Table I), although no significant differences were seen when compared to ACA negative SS patients. HLA-DR1, and to a lesser degree HLA-DR4 and DRw8, were also more frequent, but not significantly so. In fact, HLADR1, DR4, DR5(DRw11) and/or DRw8 occurred in 93\% of ACA positive patients compared to $68 \%$ of ACA negative SS patients $(P=0.003, \mathrm{OR}=6.4)$ and $58 \%$ of controls $(P=1.3$ $\times 10^{-4}$, OR $=9.6$ ). Also seen was a decreased frequency of HLA-DR3(DRw17) which was only significant when compared to ACA negative SS patients $(P c=0.04)$.

Given the weak association of ACA with HLADR5(DRw11), as well as previous studies showing correlations of ACA with HLA-DR1 and DR4 (29), oligotyping for HLADRB1 alleles was performed on all HLA-DR1, DR4 and DR5(DRw11) positive patients and controls. The frequencies of DRB1*0101, *0102 and *0103(DR1), and DRB1*1101, $* 1102, * 1103$, and $* 1104(\mathrm{DR} 5)$, were equivalent in ACA positive and negative SS patients and controls (data not shown). On the other hand, the HLA-Dw13 subtype of DR4, including the 
Table I. HLA-DR and DQ Specificities in Caucasian SS Patients with and without $A C A$ and Controls

\begin{tabular}{lccc}
\hline \multicolumn{1}{c}{ HLA } & $\begin{array}{c}\text { ACA pos SS } \\
(\boldsymbol{n}=\mathbf{4 2})\end{array}$ & $\begin{array}{c}\text { ACA neg SS } \\
(\boldsymbol{n}=\mathbf{7 4})\end{array}$ & $\begin{array}{r}\text { Controls } \\
(\boldsymbol{n}=79)\end{array}$ \\
\hline DR1 & $43 \%$ & $23 \%$ & $27 \%$ \\
DR2 $(15,16)$ & $19 \%$ & $23 \%$ & $30 \%$ \\
DR3 (17) & $7 \% *$ & $34 \%$ & $20 \%$ \\
DR4 & $33 \%$ & $27 \%$ & $27 \%$ \\
DR5 (11) & $31 \%^{*}$ & $23 \%$ & $10 \%$ \\
DR5 (12) & $0 \%$ & $1 \%$ & $0 \%$ \\
DR6 (13) & $11 \%$ & $15 \%$ & $28 \%$ \\
DR6 (14) & $5 \%$ & $12 \%$ & $10 \%$ \\
DR7 & $7 \%$ & $20 \%$ & $19 \%$ \\
DRw8 & $17 \%$ & $5 \%$ & $6 \%$ \\
DR9 & $0 \%$ & $4 \%$ & $3 \%$ \\
DRw10 & $0 \%$ & $0 \%$ & $1 \%$ \\
DQw2.1 & $7 \% *$ & $34 \%$ & $20 \%$ \\
DQw2.2 & $5 \%$ & $18 \%$ & $15 \%$ \\
DQw4 & $10 \%$ & $4 \%$ & $5 \%$ \\
DQw5 & $52 \%^{8}$ & $31 \%$ & $32 \%$ \\
DQw6 & $26 \% "$ & $38 \%$ & $54 \%$ \\
DQw7 & $55 \% 1$ & $34 \%$ & $32 \%$ \\
DQw8 & $19 \%$ & $23 \%$ & $16 \%$ \\
DQw9 & $5 \%$ & $5 \%$ & $8 \%$ \\
& & & \\
\hline
\end{tabular}

* $P c=0.04$ compared to ACA negative SS patients.

${ }^{\ddagger} P=0.09, \mathrm{OR}=4$ compared to controls.

${ }^{8} P<0.04$ (uncorrected), OR $=2.5$ compared to ACA negative SS patients or controls.

" $P \mathrm{c}=0.04$ compared to controls.

${ }^{\prime} P=0.02, \mathrm{OR}=2.6$ compared to controls.

DRB 1*0403 and *0407 alleles (defined by the presence of a glutamic acid residue at position 74 of the DRB1 first domain), was increased in frequency in those Caucasians with ACA $(19 \%)$ compared to ACA negative SS patients $(3 \%)(P c=0.05$, $\mathrm{OR}=7.6)$ and controls $(4 \%)(P c=0.08, \mathrm{OR}=6)$. In fact, HLA-Dw13 accounted for over half of the HLA-DR4 DRB1 alleles in the ACA positive patients, compared to $11 \%$ of ACA negative PSS patients $(P=0.02$, uncorrected), OR $=9.7)$ and $9 \%$ of controls $(P c=0.005$, OR $=11)$. HLA-Dw14 (DRB1*0404, ${ }^{*} 0408$ ), the HLA-DR4 subtype which shares an identical third hypervariable region sequence with HLA-DR 1, was notably, although not significantly, decreased in frequency in the ACA positive SS patients.

Determination of HLA-DQ specificities showed HLA$\mathrm{DQw} 5$ and $\mathrm{DQw} 7$ to be slightly increased in frequency compared to ACA negative SS patients and controls $(P<0.04$, uncorrected), $\mathrm{OR}=2.5$ for $\mathrm{DQw5} ; P=0.02, \mathrm{OR}=2.6$ for DQw7) (Table I). It should be noted that HLA-DQw5 is in linkage disequilibrium with HLA-DR 1, and HLA-DQw7 with HLA-DR5 and some HLA-DR4 haplotypes. It is also noteworthy that 11 of 18 DR 4 haplotypes (at least 4 of the 8 having Dw1 3 and 6 of the 9 having Dw4) in the ACA positive patients were associated with $\mathrm{DQw} 7$ (as opposed to $\mathrm{DQw8}$ ) compared to 3 of 21 (all associated with DR4[Dw4]) in the ACA negative PSS patients $(P=0.007, \mathrm{OR}=9.4)$. Two additional ACA positive patients had both DR4(Dw4) and DR4(Dw13) as well as both $\mathrm{DQw} 7$ and DQw8, thus haplotype associations could not be determined in them. In contrast, the Dw14(DQB $1^{*} 0404$,
*0408) subtype of DR4, which was rarely seen in the ACA positive patients, was always associated with $\mathrm{DQw8}$.

In order to test the relative strengths of the associations of HLA-DR5(DRw11) and DQw7 with the ACA response, we tested the presence vs. absence of one of the HLA-specificities in patients and controls in individuals with and without the other HLA-specificity, as proposed by Svejgaard and Ryder (51). All HLA-DR5 (DRw11) positive Caucasian patients and controls also had HLA-DQw7. However, in DR5 (DRw11) negative individuals, DQw7 occurred in 34\% of ACA positive patients, compared with $16 \%$ of ACA negative SS patients $(P$ $=0.08)$ and $24 \%$ of controls $(P=0.4)$. We also found HLA$\mathrm{DQw} 7$ to be associated with the antitopoisomerase I response (data not shown, manuscript in preparation). Accordingly, if anti-topoisomerase I positive SS patients were considered separately, the frequency of HLA-DQw7 in the DR5(DRw11) negative, ACA negative group of SS patients fell to $11 \%(P=0.055$ compared to ACA positive patients), suggesting the HLADQw7 association with ACA to be independent of HLADR5(DRw11).

Typing for HLA-DQA1 alleles (Table II) showed only a weak increase of frequency of DQA $1^{*} 0101$, found on most HLA-DQw5 heterodimers, compared to ACA negative SS patients and controls $(P<0.04$, uncorrected, $\mathrm{OR}=2.4)$. HLA$\mathrm{DQB1}$ allele determinations showed an increase in the frequency of DQB1*0301 (found only on DQw7 haplotypes) compared to controls $(P=0.02, \mathrm{OR}=2.6)$. HLA-DQB $1 * 0501$

Table II. HLA-DQA1 and DQB1 Alleles in ACA Positive and Negative Caucasian SS Patients and Controls

\begin{tabular}{|c|c|c|c|}
\hline Allele & $\begin{array}{c}\text { ACA pos SS } \\
(n=42)\end{array}$ & $\begin{array}{c}\text { ACA neg SS } \\
(n=74)\end{array}$ & $\begin{array}{l}\text { Controls } \\
(n=79)\end{array}$ \\
\hline DQA $1 * 0101$ & $52 \% *$ & $31 \%$ & $32 \%$ \\
\hline $\mathrm{DQA} 1 * 0102$ & $28 \%$ & $28 \%$ & $34 \%$ \\
\hline DQA $1 * 0103$ & $7 \%$ & $8 \%$ & $20 \%$ \\
\hline DQA $1 * 0201$ & $7 \%$ & $18 \%$ & $19 \%$ \\
\hline DQA1 $* 0301$ & $35 \%$ & $30 \%$ & $34 \%$ \\
\hline DQA $1 * 0401$ & $12 \%$ & $5 \%$ & $5 \%$ \\
\hline DQA $1 * 0501$ & $38 \%^{\ddagger}$ & $61 \%$ & $37 \%$ \\
\hline DQB $1 * 0201$ & $12 \%^{\S}$ & $50 \%$ & $33 \%$ \\
\hline $\mathrm{DQB} 1 * 0301$ & $55 \% "$ & $34 \%$ & $32 \%$ \\
\hline $\mathrm{DQB} 1 * 0302$ & $19 \%$ & $23 \%$ & $16 \%$ \\
\hline $\mathrm{DQB} 1 * 0303$ & $5 \%$ & $5 \%$ & $8 \%$ \\
\hline DQB $1 * 0402$ & $10 \%$ & $4 \%$ & $5 \%$ \\
\hline $\mathrm{DQB} 1 * 0501$ & $45 \%^{\prime}$ & $24 \%$ & $27 \%$ \\
\hline $\mathrm{DQB} 1 * 0502$ & $2 \%$ & $1 \%$ & $4 \%$ \\
\hline DQB $1 * 0503$ & $7 \%$ & $7 \%$ & $5 \%$ \\
\hline $\mathrm{DQB} 1 * 0601$ & $5 \%$ & $1 \%$ & $4 \%$ \\
\hline DQB $1 * 0602$ & $14 \%$ & $19 \%$ & $25 \%$ \\
\hline $\mathrm{DQB} 1 * 0603$ & $2 \%$ & $5 \%$ & $16 \%$ \\
\hline DQB $1 * 0604$ & $5 \%$ & $11 \%$ & $9 \%$ \\
\hline
\end{tabular}

* $P<0.04$ (uncorrected), OR $=2.4$ compared to either ACA negative SS or controls.

${ }^{\ddagger} P=0.03$ (uncorrected) compared to ACA negative SS.

${ }^{8} P \mathrm{c}=0.001$ compared to ACA negative SS.

" $P=0.02, \mathrm{OR}=2.6$ compared to controls.

' $P=0.02$ (uncorrected), $\mathrm{OR}=2.4$ compared to ACA negative $\mathrm{SS}$ and controls. 
(found on most DQw5 haplotypes) was slightly increased in the ACA positive SS patient compared to ACA negatives and controls, though only significantly so when the two latter groups were considered together $(P=0.04$ [uncorrected], $\mathrm{OR}=2.2)$.

Oligotyping for 19 DPB1 alleles in 35 Caucasian ACA positive patients, 54 ACA negatives and 62 controls showed equivalent frequencies in all groups studied (52).

Examination of HLA-DRB1 first domain sequences (53) showed that HLA-DR1, DR4(Dw13), DR5(DRw11) and DRw8 do not share any unique amino acid residues (or segments thereof). Similarly, there was no unique HLA-DQA1 amino acid residue, or segment of amino acids, that accounted for all (or even a majority of) ACA positive SS patients compared to ACA negative SS patients or controls.

All but three of the 42 ACA positive SS patients had HLADQB1 chains comprising DQw5 and/or DQw7. The DQB1 chains associated with HLA-DQw5 and DQw7 lack the hydrophobic residue leucine (Leu) at position 26 of the DQB1 first domain (54), located in the floor of the putative peptide-binding groove in the second hypervariable region (55). Instead, these DQB1 alleles have either tyrosine or glycine at this position, both polar non-charged amino acids. Furthermore, the three ACA positive patients lacking DQw5 and DQw7 had $\mathrm{DQB} 1{ }^{*} 0601$ or ${ }^{*} 0402$ alleles, which also have tyrosine or glycine, respectively, at position 26 . In total, therefore, the presence of one or more of these non-Leu-26 DQB1 alleles accounted for $100 \%$ of the Caucasian ACA positive patients, compared to $69 \%$ of the ACA negative SS patients $(P=0.0008)$ and $71 \%$ of the controls $(P=0.0003)$. This association was further underscored by examining genotype frequencies of non-Leu-26 DQB1 alleles, where non-Leu-26 alleles accounted for $73 \%$ of 84 HLA-DQB1 genotypes in ACA patients compared to $42 \%$ of 148 in ACA negative PSS patients $(P=1.2$ $\left.\times 10^{-5}\right)$ and $38 \%$ of 158 in controls $\left(P=5.8 \times 10^{-7}\right)$. In fact, $43 \%$ of ACA positive SS patients had both of their DQB1 alleles lacking Leu-26, compared to 7\% of ACA negative SS patients $\left(P=1 \times 10^{-4}\right)$ and $14 \%$ of controls $\left(P=4 \times 10^{-4}\right)$ (Table III). This might suggest a gene dosage effect, however homozygosity for these DQB1 alleles occurred no more frequently than would be expected by Hardy Weinberg equilibrium ( $43 \%$ vs $50 \%, P=\mathrm{NS})$. The association with non-Leu-26 alleles persisted even when the HLA-DR5(DRw11) positive individuals were removed, being present in $100 \%$ of the remaining ACA positive SS patients compared with 59\% of DR 5 negative, ACA negative SS patients $\left(P=1.6 \times 10^{-4}\right)$ and $59 \%$ of DR5 negative controls $\left(P=1.2 \times 10^{-4}\right)$.

As far as any possible protective role against developing ACA for the presence of leucine at position 26 of the DQB1 first domain is concerned, it should be noted that the frequency of having one non-Leu- 26 allele was the same $(57 \%-61 \%)$ in all groups studied, making this unlikely.

Regional differences in HLA-class II gene frequencies could at least in part explain the marked predominance of certain HLA-DQ alleles in ACA positive versus negative SS patients and controls, especially because the Caucasian controls here examined by RFLP and oligotyping were Houston medical students and health care workers of preponderantly Northern European ancestry. In fact, the frequency of HLA-DQw5 and DQw7 (determined by RFLP only) among 83 Caucasian controls from Ottawa was nearly twice that seen in Houston ( $R$. Goldstein, unpublished data). HLA-class II gene frequencies from Miami controls were not available. However, the fre-
Table III. Frequency of Homozygosity and Heterozygosity for HLA-DQBI Alleles Lacking Leucine at Position 26 (Leu-26 negative) in Predisposition to the ACA Response in Caucasians

\begin{tabular}{lccc}
\hline & $\begin{array}{c}\text { ACA pos SS } \\
(n=42)\end{array}$ & $\begin{array}{c}\text { ACA neg SS } \\
(n=74)\end{array}$ & $\begin{array}{c}\text { Controls } \\
(n=79)\end{array}$ \\
\hline Both DQB1 alleles Leu-26 negative & $43 \%^{*, *}$ & $7 \%$ & $14 \%$ \\
One DQB1 allele Leu-26 negative & $57 \%$ & $61 \%$ & $57 \%$ \\
Both DQB1 alleles Leu-26 positive & $0 \%^{8.11}$ & $32 \%$ & $29 \%$ \\
\hline
\end{tabular}

* $P=1 \times 10^{-4} ;{ }^{8} P=8 \times 10^{-4}$ compared to ACA neg SS.

₹ $P=4 \times 10^{-4} ; "$ " $P=3 \times 10^{-4}$ compared to normal controls.

quencies of Leu-26 negative DQB1 alleles between ACA positive and negative SS patients was similar in all three regions. The most significant differences were seen between the larger numbers of ACA positive $(n=20)$ and negative $(n=30)$ Caucasian SS patients from Houston, where the non-Leu-26 DQB1 genotypic frequencies were $80 \%$ vs $36 \%\left(P=4 \times 10^{-5}, \mathrm{OR}=7\right)$. Among those from Ottawa (14 ACA positive, 19 ACA negative) the genotype frequencies were $64 \%$ vs $34 \%(P=0.03$, OR $=3.5$ ). The number of ACA positive patients from Miami was small $(n=5)$ vs those ACA negative, $(n=22)$. Nevertheless, the frequency of non-Leu-26 DQB1 alleles was $80 \%$ vs $48 \%$ ( $P$ $=0.086, \mathrm{OR}=4.4)$. HLA-DQw5 and DQw7 were more frequent in ACA positive versus negative SS patients from all regions, although the differences did not achieve statistical significance, perhaps due to the smaller numbers examined.

All of seven other ACA positive patients of non-Caucasian ethnic origin also possessed non-Leu-26 DQB1 alleles, the majority having two. The one American black SS patient with ACA had HLA-DR5(DRw11), DQw7; DRw8, DQw4. Two additional American blacks with polymyositis also had ACA, one having HLA-DR1, DQw5; DR4(Dw13), DQw7 and the other DR1, DQw5; DRw8, DQw7. The Hispanic SS patient with ACA had DR1, DQw5 and DR4(Dw13), DQw8, as did both an ACA positive Hispanic with Sjögren's syndrome and another with dermatomyositis. A Chinese SS patient had HLADRw10, DQw5; DRw14, DQw5.

\section{Discussion}

Previous studies have examined HLA-DR specificities in SS patients with the ACA response, finding associations with the broad specificities HLA-DR1 (8, 29), DR4 (29), DR5(DRw11) (23), and possibly DRw8 (29). Our earlier preliminary data, examining smaller numbers of patients than in this study, suggested weak associations of ACA with HLA-DR1 and DR4 (56). The amino acid sequence shared by DR1 and the Dw14 (DRB1*0404, *0408) subtype of DR4, (QRRAA), which comprises residues 71-74 in the third hypervariable region of DRB1, was postulated to form an "epitope" predisposing to this autoantibody response. Subsequent DR4 subtyping, however, showed that only the Dw13(DRB1*0403, *0407) subtype was increased in frequency, and, in fact, that Dw14(DRB 1*0404, ${ }^{*} 0408$ ) was less common in the ACA positive SS patients. HLA-DR4(Dw13) has a glutamic acid residue at position 74 of the DRB1 first domain, which is negatively 
charged, whereas DR1 and DR4(Dw14) have an alanine, which is a hydrophobic residue, at this position. Thus DR 1 and DR4(Dw13) would be expected to have significant physiochemical differences in this segment of the third hypervariable region, which could have a major impact on antigen and/or $T$ cell receptor interactions. Furthermore, 39\% of the ACA positive patients lacked DR 1 or DR4, with nearly all the remaining patients having DR5(DRw11) and/or DRw8. HLA-DR1, DR4, DR5(DRw11) and DRw8 share no common unique DRB1 epitope. Therefore, if one is to explain the ACA response by a unique HLA class II amino acid (or sequence thereof), DRB1 does not appear to be the candidate locus.

Furthermore, no HLA-DRB3(DRw52) or DRB4(DRw53) association was found for the ACA response (data not shown). Also, of the 19 DPB1 alleles examined, none were increased in frequency in either $\mathrm{SS}$ or the ACA response. Despite a marginal increase in DQA1*0101 (found on most DQw5-bearing haplotypes), no DQA1 sequence or amino acid discriminated ACA positive individuals.

By themselves, the $\mathrm{DQB} 1$ alleles comprising $\mathrm{DQw} 5$ as well as $\mathrm{DQw} 7\left(\mathrm{DQB} 1{ }^{*} 0301\right)$ were only marginally increased, but together they accounted for most of the ACA positive SS patients, with DQB1*0402 and *0601 accounting for the rest. HLA-DR5(DRw11) and many DR4(Dw13) and DR4(Dw4) haplotypes are in linkage disequilibrium with DQw7(DQB1*0301), which may account for their association with ACA. On the other hand, the DQB1 chains comprising DQw6 and $D Q w 2\left(D Q B 1{ }^{*} 0201\right)$ were less frequent in SS patients with ACA. These ACA-associated and nonassociated DQB1 alleles differ at position 26 of the first domain, in the floor of the antigen binding cleft in the second hypervariable region. The presence of a polar tyrosine or glycine residue was specifically associated with the ACA response, whereas the presence of a hydrophobic leucine here was not. Still, $11 \%$ of ACA negative SS patients had both of their DQB1 alleles lacking leucine at position 26. While the reason these patients lacked ACA autoantibodies is not clear, it can be speculated that their ACA level was below that which was detectable by the immunofluorescence assay here utilized, or that perhaps they lacked the appropriate T cell receptor genes to which these MHC class II heterodimers could present the processed centromere autoantigenic peptide. Furthermore, all non-Caucasians with ACA had nonLeu-26 DQB1 alleles. This transracial association further suggests the importance of this amino acid residue in the ACA response.

We have found position 26 of the DQB1 chain to be associated with other autoantibody responses. The anti-Ro(SS-A) response in patients with systemic lupus erythematosus and Sjögren's syndrome has as a minimal requirement the presence of the hydrophobic leucine here (and/or a glutamine at position 34 of the DQA1 chain) (57). Thus it seems that charge (hydrophobicity vs. hydrophilicity) at this position in the putative antigen binding cleft may influence the specificity of the autoantibody response.

Therefore the HLA-DR5 (DRw11) association with SS per se would appear to be explained, at least in part, by linkage disequilibrium with HLA-DQ genes, themselves associated with the ACA response. Data from our laboratory also suggests the antitopoisomerase (anti-Scl-70) response to be correlated with different DQB1 loculs epitopes (manuscript in preparation). Thus, HLA-class II associations with SS may be better explained by autoantibody subsets of this disease.

\section{Acknowledgments}

The authors wish to thank Miriam MacLeod-St. Clair, Kent Anderson, and Nancy Biggs for technical assistance, and Grace L. Griffin for preparation of this manuscript.

Supported by a grant from the Scleroderma Research Foundation (1094), and a grant from the Scleroderma Foundation, Inc. (R. Goldstein), as well as by National Institutes of Health grants AR39325 (J. D. Reveille) and DK-39965 (D. Owerbach) and an Arthritis Foundation Clinical Research Center Grant. Dr. Reveille is an Investigator of the Arthritis Foundation. Dr. Goldstein is a Career Scientist of the Ontario Ministry of Health, Health Research Personnel Development Program. The results and conclusions are those of the authors and no official endorsement by the Ontario Ministry of Health is intended or should be inferred.

\section{References}

1. Fritzler, M. J., J. D. Kinsella, and E. Garbutt. 1980. The CREST syndrome: a distinct serologic entity with anticentromere antibodies. Am. J. Med. 69:520526.

2. Tan, E. M., G. P. Rodnan, I. Garcia, Y. Moroi, M. J. Fritzler, and C. Peebles. 1980. Diversity of antinuclear antibodies in progressive systemic sclerosis: anti-centromere antibody and its relationship to CREST syndrome. Arthritis Rheum. 23:617-625.

3. Kallenberg, C. G. M., G. W. Pastoor, A. A. Wouda, and T. H. The. 1982. Antinuclear antibodies in patients with Raynaud's phenomenon: clinical signifcance of anticentromere antibodies. Ann. Rheum. Dis. 41:382-387.

4. Catoggio, L. J., R. P. Skinner, and P. J. Maddison. 1983. Frequency and clinical significance of anticentromere and anti-Scl-70 antibodies in an English connective tissue disease population. Rheumatol. Int. 3:19-21.

5. Steen, V. D., G. L. Ziegler, G. P. Rodnan, and T. A. Medsger, Jr. 1984. Clinical and laboratory associations of anticentromere antibody in patients with progressive systemic sclerosis. Arthritis Rheum. 27:125-131.

6. Nishikai, M., Y. Okano, H. Yamashita, and M. Watanabe. 1984. Characterization of centromere (kinetochore) antigen reactive with sera of patients with a scleroderma variant (CREST syndrome). Ann. Rheum. Dis. 43:819-824.

7. Weiner, E. S., W. C. Earnshaw, J. L. Senécal, B. Bordwell, P. Johnson, and N. F. Rothfield. 1988. Clinical associations of anticentromere antibodies and antibodies to topoisomerase I: a study of 355 patients. Arthritis Rheum. 31:378385.

8. Steen, V. D., D. L. Powell, and T. A. Medsger Jr. 1988. Clinical correlations and prognosis based on serum autoantibodies in patients with progressive systemic sclerosis. Arthritis Rheum. 31:196-203.

9. Shero, J. H., B. Bordwell, N. F. Rothfield, and W. C. Earnshaw. 1986. High titers of autoantibodies to topoisomerase I (Scl-70) in sera of scleroderma patients. Science (Wash. DC). 231:737-740.

10. Reimer, G., V. D. Steen, C. A. Penning, T. A. Medsger Jr., and E. M. Tan. 1988. Correlates between autoantibodies to nucleolar antigens and clinical features in patients with systemic sclerosis (scleroderma). Arthritis Rheum. 31:525532.

11. Clements, P. J., G. Opelz, P. I. Terasaki, M. R. Mickey, and D. Furst. 1978. Association of HLA antigen A9 with progressive systemic sclerosis (scleroderma). Tissue Antigens. 11:357-361.

12. Ercilla, M. G., F. Arriaga, M. R. Gratacos, J. Coll, V. Lecha, J. Vives, and R. Castillo. 1981. HLA antigens and scleroderma. Arch. Dermatol. Res. 271:381385.

13. Hughes, P., K. Gelsthorpe, R. W. Doughty, N. R. Rowell, F. D. Rosenthal, and I. B. Sneddon. 1978. The association of HLA-B8 with visceral disease in systemic sclerosis. Clin. Exp. Immunol. 31:351-356.

14. Kallenberg, C. G. M., J. M. van der Voort-Beelen, J. d'Amaro, and T. H. The. 1981. Increased frequency of B8/DR3 in scleroderma and association with impaired cellular immune response. Clin. Exp. Immunol. 43:478-485.

15. Mollenhauer, E., R. Schmidt, M. Heinrichs, and C. Rittner. 1984. Scleroderma: possible significance of silent alleles at the C4B locus. Arthritis Rheum. 27:711-712.

16. Lynch, C. J., G. Singh, T. L. Whiteside, G. P. Rodnan, T. A. Medsger, and B. S. Rabin. 1982. Histocompatibility antigens in progressive systemic sclerosis (PSS; scleroderma). J. Clin. Immunol. 2:314-318.

17. DiBartolomeo, A. G., B. S. Rabin, and G. P. Rodnan. 1981. HLA-D antigens in progressive systemic sclerosis (Scleroderma). Immunol. Commun. 10:733-740.

18. Black, C. M., K. I. Welsh, P. J. Maddison, M. I. V. Jayson, and R. M. Berstein. 1984. HLA antigens, autoantibodies and clinical subsets in scleroderma. Br. J. Rheumatol. 23:267-271. 
19. Alarcon, G. S., R. M. Phillips, C. K. Wasner, R. T. Acton, and B. O. Barger. 1985. DR antigens in systemic sclerosis: lack of clinical correlations Tissue Antigens. 26:156-158.

20. Germain, B. F., L. R. Espinoza, L. L. Bergen, M. Vagesh, and F. B. Vasey. 1981. Increased prevalence of DRw3 in the CREST syndrome. Arthritis Rheum. 24:857-859.

21. Gladman, D. D., E. C. Keystone, M. Baron, P. Lee, D. Cane, and H. Mervert. 1981. Increased frequency of DR5 in scleroderma. Arthritis Rheum. 24:854-856.

22. Briggs, D. C., K. Welsh, R. S. Pereira, and C. M. Black. 1986. A strong association between null alleles at the $\mathrm{C} 4 \mathrm{~A}$ locus in the major histocompatibility complex and systemic sclerosis. Arthritis Rheum. 29:1274-1277.

23. Dunckley, H., E. C. Jazwinska, P. A. Gatenby, and S. W. Serjeantson. 1989. DNA-DR typing shows HLA-DRw1 1 RFLPs are increased in frequency in both progressive systemic sclerosis and CREST variants of scleroderma. Tissue Antigens. 33:418-420.

24. Rabin, B. S., G. P. Rodnan, S. Bassion, and T. J. Gill. 1975. HLA antigens in progressive systemic sclerosis (scleroderma). (Letter.) Arthritis Rheum. 18:381-382.

25. Crouzet, J., M. C. Marbach, J. P. Camus, P. Godeau, G. Herreman, D. Richier, J. Hors, and J. Dausset. 1975. Recherche d'une association entre antigens HL-A et sclerodermie systemique. Presse Med. 4:2489-2492.

26. Birnbaum, N. S., G. P. Rodnan, B. S. Rabin, and S. Bassion. 1977. Histocompatibility antigens in progressive systemic sclerosis (scleroderma). J. Rheumatol. 4:425-428.

27. Niks, M., J. Rovensky, J. Nyulassy, M. Buc, J. Stefanovic, and D. Zitnan. 1982. Lack of association of HLA-DR antigens with progressive systemic sclerosis (scleroderma). Tissue Antigens. 19:238-239.

28. Livington, J. Z., T. E. Scott, F. M. Wigley, G. J. Anhalt, W. B. Bias, R. H McLean, and M. C. Hochberg. 1987. Systemic sclerosis (scleroderma): clinical, genetic and serologic subsets. J. Rheumatol. 14:512-518.

29. Genth, E., R. Mierau, P. Genetzky, C. A. von Mühlen, S. Kaufman, H von Wilmowsky, M. Meurer, T. Krieg, H. J. Pollman, and P. W. Hartl. 1990 Immunogenetic associations of scleroderma-related antinuclear antibodies. $\mathrm{Ar}$ thritis Rheum. 33:657-665.

30. Pereira, S., C. Black, K. Welsh, B. Ansell, M. Jayson, P. Maddison, and N. Rowell. 1987. Autoantibodies and immunogenetics in 30 patients with systemic sclerosis and their families. J. Rheumatol. 14:760-765.

31. Arnett, F. C., W. B. Bias, R. H. McLean, M. Engel, M. Duvic, R. Goldstein, L. Freni-Titulaer, T. W. McKinley, and M. C. Hochberg. 1990. Connective tissue disease in southeast Georgia. A community based study of immunogenetic markers and autoantibodies. J. Rheumatol. 17:1029-1035.

32. Subcommittee for Scleroderma Criteria of the American Rheumatism Association Diagnostic and Therapeutic Criteria Committee. 1980. Preliminary criteria for the classification of systemic sclerosis (scleroderma). Arthritis Rheum. 23:581-590.

33. Blin, N., and D. W. Stafford. 1978. A general method for isolation of high molecular weight DNA from eukaroytes. Nucleic Acids Res. 3:2303-2308.

34. McDaniel, D. O., R. T. Acton, B. O. Barger, W. J. Koopman, and J. D. Reveille. 1987. Association of 9.2 kilobase PvulI class I major histocompatibility complex restriction fragment length polymorphism with ankylosing spondylitis. Arthritis Rheum. 30:894-900.

35. Long, E. O., C. T. Wake, J. Gorski, and B. Mach. 1983. Complete sequence of an HLA-DR $\beta$ chain gene. EMBO (Eur. Mol. Biol. Organ.) J. 2:389394.

36. Larhammar, D., J. J. Hyldig-Nielsen, B. Servenius, G. Anderson, L. Rask and P. A. Peterson. 1983. Exon-intron organization and complete nucleotide sequence of a human major histocompatibility antigen $\mathrm{DC} \beta$ gene. Proc. Natl. Acad. Sci. USA. 80:7313-7317.

37. Spielman, R. S., J. Lee, W. F. Bodmer, J. G. Bodmer, and J. Trowsdale. 1984. Six HLA-D region $\alpha$-chain genes on human chromosome 6: polymorphisms and associations of DQ $\alpha$-related sequences and DR types. Proc. Natl. Acad. Sci. USA. 81:3461-3465.
38. Feinberg, A. P., and B. Vogeistein. 1983. A technique for radiolabelling DNA restriction fragments to high specific activity. Anal. Biochem. 132:6-13.

39. Cohen, D., M. Simons, J. M. Lalouel, and B. DuPont. 1988. Nomenclature for HLA-RFLP. Tenth International Histocompatibility Workshop Newsletter. 2:44-51.

40. Reveille, J. D., R. R. Schrohenloher, R. T. Acton, and B. O. Barger. 1989. DNA analysis of HLA-DR and DQ genes in American blacks with systemic lupus erythematosus. Arthritis Rheum. 32:1243-1251.

41. Reveille, J. D., R. T. Acton, K. Anderson, B. O. Barger, and R. E. Schrohenloher. 1990. RFLP analysis of HLA-DR, DQ, DP and C4 alleles in Caucasians with systemic lupus erythematosus. J. Rheumatol. 18:14-18.

42. Kohonen-Corish, M. R. J., and S. W. Serjeantson. 1986. HLADR $\beta$ polymorphism revealed by Taq I correlate with HLA-DR specificities. Hum Immunol. 15:263-271.

43. Carlsson, B., J. Wallin, J. Bohme, and E. Moller. 1987. HLA-DR-DQ haplotypes defined by restriction fragment analysis: correlation to serology. Hum. Immunol. 20:95-113.

44. MacMurray, A. J., J. I. Bell, D. Denney, D. Watling L. S. Foster, and H. O. McDevitt. 1987. Molecular mapping of class II polymorphisms in the human major histocompatibility complex. II. DQß. J. Immunol. 139:574-586.

45. Bell, J. I., D. Denney, A. MacMurray, L. Foster, D. Watling, and H. O McDevitt. 1987. Molecular mapping of class II polymorphisms in the human major histocompatibility complex. I. DR $\beta$. J. Immunol. 139:562-573.

46. Bidwell, J. L., E. A. Bidwell, D. A. Savage, D. Middleton, P. T. Klouda, and B. A. Bradley. 1988. A DNA-RFLP typing system that positively identifies serologically well defined and ill defined HLA-DR and DQ alleles, including DRw10. Transplantation (Baltimore). 45:640-646.

47. Bodmer, J. G., S. G. E. Marsh, P. Parham, H. A. Erlich, E. Albert, W. F Bodmer, B. Dupont, B. Mach, W. R. Mayr, T. Sasazuki, G. M. T. Schreuder, J. L. Strominger, A. Svejgaard, and P. I. Terasaki. 1990. Nomenclature for factors of the HLA system. 1989. Hum. Immunol. 28:326-342.

48. Moraes, M. E., M. Fernandez-Vina and P. Stastny. 1991. DNA typing for Class II HLA antigens with allele-specific or group specific amplification. IV. Typing for alleles of the HLA-DR2 group. Hum. Immunol. 31:139-144.

49. Owerbach D., S. Gunn, and K. H. Gabbay. 1989. Primary association of HLA-DQw8 with type I diabetes in DR4 patients. Diabetes. 38:942-949.

50. Gao, X., M. Fernandez-Vina, W. Shumway, and P. Stastny. 1990. DNA typing for class II HLA antigens with allele-specific or group-specific amplification I. Typing for subsets of HLA-DR4. Hum. Immunol. 27:40-50.

51. Svejgaard, A. and L. P. Ryder. 1977. Associations between HLA and disease. Notes on methodology and a report from the HLA and Disease Registry. In HLA and Disease, J. Dausset and A. Svejgaard, editors. William and Wilkins Co., Baltimore, Maryland. 46-54.

52. Reveille, J. D., J. Brady, M. MacLeod-St. Clair, and E. Durban. 1992. HLA-DPB 1 alleles and autoantibody subsets in systemic lupus erythematosus, Sjögren's syndrome and progressive systemic sclerosis. A question of disease relevance. Tissue Antigens. In press.

53. Marsh, S. G. E., and J. G. Bodmer. 1990. HLA-DRB nucleotide sequences. Immunogenetics. 31:141-144.

54. Gregersen, P. K., J. A. Todd, H. A. Erlich, E. Long, B. Servenius, E. Choi, H. T. Kao, and J. S. Lee. 1989. First domain sequence diversity of DR and DQ subregion alleles. In Histocompatibility Testing 1987, Vol. I. Immunobiology of HLA. B. Dupont, editor. Springer-Verlag, New York, New York. 1027-1034

55. Brown, J. H., T. Jardetzky, M. Saper, B. Samraoui, P. Bjorkman, and D. C. Wiley. 1988. A hypothetical model of the foreign antigen binding site of class II histocompatibility molecules. Nature (Lond.). 332:6012-6015.

56. Isern, R., F. Arnett, R. Goldstein, K. Anderson, E. Durban, D. Owerbach, and J. Reveille. 1990. Anti-centromere antibodies (ACA) are associated with DR $\beta 1$ third hypervariable sequences shared by HLA-DR1 and DR4. Arthritis Rheum. 33(Suppl.):S32.

57. Reveille, J. D., M. J. MacLeod, K. Whittington, and F. C. Arnett. 1991. Specific amino acid residues in the second hypervariable region of HLA-DQA 1 and DQB1 chain genes promote the Ro (SS-A)/La (SS-B) autoantibody responses. J. Immunol. 146:3871-3876. 